\title{
On Plücker coordinates of a perfectly oriented planar network
}

\author{
Kelli Talaska \\ Department of Mathematics, University of Michigan, Ann Arbor, MI 48109, USA
}

\begin{abstract}
Let $G$ be a perfectly oriented planar graph. Postnikov's boundary measurement construction provides a rational map from the set of positive weight functions on the edges of $G$ onto the appropriate totally nonnegative Grassmann cell. We establish an explicit combinatorial formula for Postnikov's map by expressing each Plücker coordinate of the image as a ratio of two polynomials in the edge weights, with positive integer coefficients. These polynomials are weight generating functions for certain subsets of edges in $G$.
\end{abstract}

Keywords: boundary measurements, total positivity, totally nonnegative Grassmannian, perfectly oriented network

Totally nonnegative Grassmannians are a subclass of totally nonnegative homogeneous spaces, first introduced and studied by G. Lusztig and K. Rietsch (see, e.g., [6, 8, 9]). Informally speaking, one takes the part of a real Grassmann manifold where all Plücker coordinates are nonnegative. A. Postnikov's groundbreaking paper [7] established combinatorial foundations for the study of totally nonnegative Grassmannians, in particular providing the tools required for the construction of cluster algebra structures in (ordinary) Grassmannians by J. Scott [10], and for the exploration of tropical analogues by D. Speyer and L. Williams [11].

In this extended abstract, we describe an explicit combinatorial formula for the main construction in [7]: the boundary measurement map assigning a point in the totally nonnegative Grassmannian to a planar directed network with positive edge weights. For the complete version of this work, including rigorous statements of the theorems, full proofs, and several generalizations, see [12]. To describe our formula, we will need to quickly recall the main features of Postnikov's construction. To illustrate the concepts, we will refer to our running example, given in Figure 1.

The construction begins with a planar directed graph $G$ properly embedded in a disk. A network $N$ is formed by assigning a formal variable weight to each edge of $G$. Let $\left\{b_{1}, \ldots, b_{n}\right\}$ be the set of boundary vertices of $G$, i.e., those lying on the boundary of the disk, labeled in clockwise order around the boundary of the disk. Assume that each boundary vertex is either a source or a sink. Postnikov defines the boundary measurement $M_{i j}$ as a weight generating function for directed walks from the boundary vertex $b_{i}$ to the boundary vertex $b_{j}$, with each walk counted with a sign reflecting the parity of its topological winding index.

The boundary measurement matrix $A$ is defined in term of these boundary measurements. Its columns are labeled by the $n$ boundary vertices, and its rows are labeled by the boundary sources. Suppose $I \subset\{1, \ldots, n\}$ indexes the set of boundary sources, and let $\Delta_{J}(A)$ denote the maximal minor of $A$ 1365-8050 (C) 2008 Discrete Mathematics and Theoretical Computer Science (DMTCS), Nancy, France 


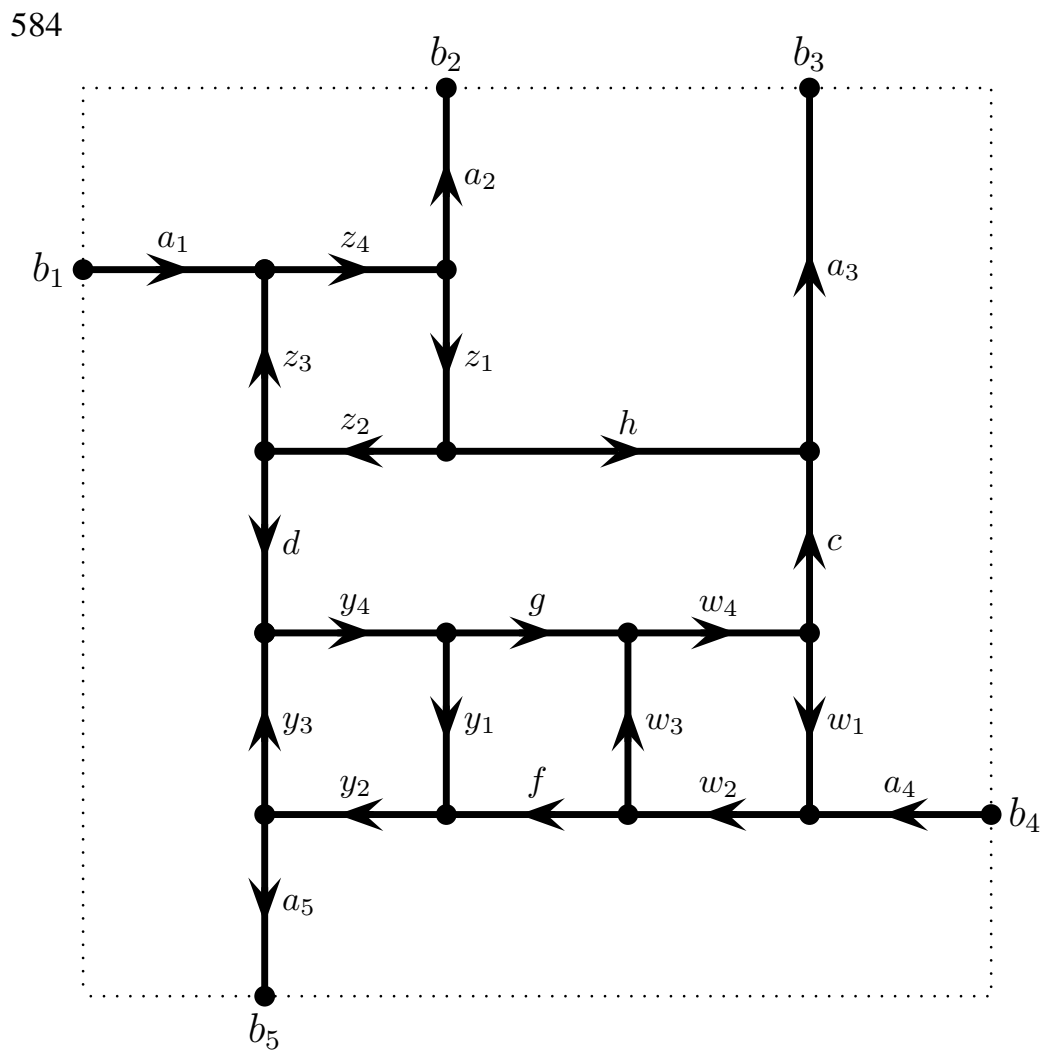

Kelli Talaska

The cycles of $N$

have weights

$W=w_{1} w_{2} w_{3} w_{4}$

$Y=y_{1} y_{2} y_{3} y_{4}$

$Z=z_{1} z_{2} z_{3} z_{4}$, and

$T=f y_{2} y_{3} y_{4} g w_{4} w_{1} w_{2}$

Fig. 1: A perfectly oriented planar directed network $N$ with boundary vertices $\left\{b_{1}, b_{2}, b_{3}, b_{4}, b_{5}\right\}$. Edges are labeled with their weights.

indexed by the column set $J$ whenever $|I|=|J|$. Then the entries of $A$ are the corresponding boundary measurements $\pm M_{i j}$, taken with signs so that the submatrix indexed by the column set $I$ is the identity matrix and $M_{i j}=\Delta_{I \backslash\{i\} \cup\{j\}}(A)$ whenever $b_{j}$ is a sink. (These requirements uniquely determine the signs.)

Example 1 Suppose that $N$ is the directed network in Figure 1. Then the boundary source set is indexed by $I=\{1,4\}$, and we have the boundary measurement matrix

$$
A=\left(\begin{array}{ccccc}
1 & M_{12} & M_{13} & 0 & -M_{15} \\
0 & M_{42} & M_{43} & 1 & M_{45}
\end{array}\right)
$$

We see that $M_{11}=M_{44}=1, M_{14}=M_{41}=0, M_{12}=\Delta_{\{2,4\}}, M_{13}=\Delta_{\{3,4\}}, M_{15}=\Delta_{\{4,5\}}$, $M_{42}=\Delta_{\{1,2\}}, M_{43}=\Delta_{\{1,3\}}$, and $M_{45}=\Delta_{\{1,5\}}$.

The maximal minors $\Delta_{J}(A)$ of the boundary measurement matrix $A$ are then interpreted as Plücker coordinates of a point in a Grassmannian. The fact that these minors are nonnegative (so that we get a point in a totally nonnegative Grassmannian) follows from the assertion in [7] that each $\Delta_{J}(A)$ can be written as a subtraction-free rational expression in the edge weights. 
Postnikov's proof of this fact is recursive. We provide a direct proof via an explicit combinatorial formula for the minors $\Delta_{J}(A)$, writing each of them as a ratio of two polynomials in the edge weights, with positive integer coefficients.

We assume that $G$ is perfectly oriented, i.e., every vertex of $G$ has exactly one incoming edge or exactly one outgoing edge (or both). There is no loss of generality in doing so, as Postnikov gives in [7] a method for transforming an arbitrary planar network into a perfectly oriented one, while preserving boundary measurements.

A simple walk is a directed walk in $G$ which does not visit any vertex more than once, except that the initial and terminal vertices may coincide. If they do, the walk is called a simple cycle. The weight of a simple walk is defined to be the product of its edge weights. A walk from a vertex to itself which has no edges is called a trivial walk and is not considered a cycle; a trivial walk has weight 1 . The weight of a collection of walks is the product of the weights of its components. (An empty collection has weight 1.)

Theorem 1 The Plücker coordinate $\Delta_{J}(A)$ is given by $\Delta_{J}=\frac{f}{g}$, where $f$ and $g$ are nonnegative polynomials in the edge weights, defined as follows:

- $f$ is the weight generating function for collections consisting of several simple cycles and $|I|$ simple or trivial walks, all of them pairwise vertex-disjoint, such that the walks connect the sources in I to the boundary vertices in $J$;

- $g$ is the weight generating function for collections of vertex-disjoint simple cycles.

Each edge set in the formula above is called a flow. Each term in the numerator is the weight of a flow from I to $J$, and each term in the denominator is the weight of a conservative flow.

Example 2 Consider the Plücker coordinate $\Delta_{J}$, with $J=\{1,5\}$ for the network $N$ in Figure 1. The boundary vertex $b_{1}$ must be connected to itself by the corresponding trivial walk. There is only one simple walk $P$ connecting the boundary vertices $b_{4}$ and $b_{5}$, but there are two flows from $\{1,4\}$ to $\{1,5\}$. The first flow consists of $P$ (and a trivial walk) and the second consists of the union of $P$ and the cycle of weight $Z=z_{1} z_{2} z_{3} z_{4}$ (and a trivial walk). Thus, the numerator is $a_{4} w_{2} f y_{2} a_{5}(1+Z)$.

The only cycles in $N$ are those of weights $W, Y, Z$, and $T$. Flows in $G$ consist of unions of disjoint cycles, so we see that the denominator is $1+W+Y+Z+T+W Z+W Y+Y Z+W Y Z+Z T=$ $(1+Z)[(1+W)(1+Y)+T]$.

Finally, we have

$$
\Delta_{J}=\frac{a_{4} w_{2} f y_{2} a_{5}(1+Z)}{(1+Z)[(1+W)(1+Y)+T]}=\frac{a_{4} w_{2} f y_{2} a_{5}}{(1+W)(1+Y)+T} .
$$

If the underlying graph $G$ is acyclic (i.e., has no oriented cycles), then the denominator $g$ in Theorem 1 is equal to 1, and the theorem reduces to the well-known result of Lindström [5] that expresses a determinant of a matrix associated with a planar acyclic network in terms of non-intersecting paths; see, e.g., [2] and references therein. Thus, Theorem 1 can be viewed as a generalization of Lindström's Lemma to nonacyclic planar networks. Another such generalization was given by S. Fomin [1], whose setup for total positivity differed from Postnikov's in that the analogues of boundary measurements did not involve any signs. In Fomin's approach, total nonnegativity is achieved by expressing the minors in question as formal power series with nonnegative coefficients in the edge weights. In contrast, Postnikov's map produces 
subtraction-free expressions which are rational (that is, involve division) but finite (that is, do not require infinite summation).

Our proof of this result is based on a sign-reversing involution in the spirit of the well-known proof of Lindström's result. Each term in $\Delta_{J}$ corresponds to a collection of walks, each taking an element of $I$ to a distinct element of $J$. By constructing an involution on pairs $(C, \mathcal{P})$ where $C$ is a collection of vertex-disjoint cycles, and $\mathcal{P}$ is such a collection of walks (which may intersect themselves or each other), we show, using the notation above, that $f=g \Delta_{J}$. The product $g \Delta_{J}$ will be a weight generating function for all pairs $(C, \mathcal{P})$. All terms corresponding to pairs in which $\mathcal{P}$ intersects $C$ or itself will cancel, leaving the weight generating function for pairs where the path collection $\mathcal{P}$ has no intersections with either itself or with the cycle collection $C$, which is precisely $f$.

Acknowledgements: The author would like to thank Alexander Postnikov for updates on the work that inspired this paper; Alek Vainshtein, Lauren Williams, and Gregg Musiker for insightful conversations; and Sergey Fomin for many helpful comments on preliminary versions of the manuscript. The author was partially supported by NSF Grants CCF-04.30201 and DMS-0502170.

\section{References}

[1] S. Fomin, Loop-erased walks and total positivity, Trans. Amer. Math. Soc. 353 (2001), 3563-3583

[2] S. Fomin and A. Zelevinsky, Total positivity: tests and parametrizations, Math. Intelligencer 22 (2000), no. 1, 23-33.

[3] G. Lawler, Intersections of Random Walks, Birkhäuser, 1991.

[4] G. Lawler, A self-avoiding random walk, Duke Math. J. 47 (1980), 655-694.

[5] B. Lindström, On the vector representations of induced matroids, Bull. London Math. Soc. 5 (1973), 85-90.

[6] G. Lusztig, Introduction to total positivity, Positivity in Lie theory: open problems, 133-145, de Gruyter Exp. Math., 26, de Gruyter, Berlin, 1998.

[7] A. Postnikov, Total positivity, Grassmannians, and networks, October 2007 version, http://math.mit.edu/ apost/tpgrass.pdf.

[8] K. Rietsch, An algebraic cell decomposition of the nonnegative part of a flag variety, J. Algebra 213 (1999), no. 1, 144-154.

[9] K. Rietsch, Closure relations for totally nonnegative cells in $G / P$, Math. Res. Lett. 13+ (2006), no. 5-6, 775-786.

[10] J. Scott, Grassmannians and cluster algebras. Proc. London Math. Soc. (3) 92 (2006), no. 2, 345380.

[11] D. Speyer and L. Williams, The tropical totally positive Grassmannian, J. Algebraic Combin. 22 (2005), no. 2, 189-210.

[12] K. Talaska, A formula for Plücker coordinates associated with a planar network, arXiv:0801.4822. 